\title{
Detection of Eccentricity Faults in Machine Using Frequency Spectrum Technique
}

\author{
Intesar Ahmed ${ }^{* a}$, Manzar Ahmed ${ }^{\mathrm{b}}$, Kashif Imran ${ }^{\text {a }}$, M. Shuja Khan **a , Member IACSIT, S. Junaid \\ Akhtar ${ }^{\text {a }}$
}

\begin{abstract}
This paper investigated the current, flux and instantaneous power signals as a diagnostic medium to detect the eccentricity faults under varying load conditions using eccentricity fault frequency components. The analysis of results based on the variations in the amplitude of sideband components between healthy and faulty motors versus average eccentricity level over a wide range of loading conditions from different signals. This study examine the usefulness of instantaneous power to detect the eccentricity faults under varying loading conditions and also shows the prime features of different signals to detect the eccentricity faults using the variations between the healthy and faulty motors when the level of average eccentricity is high and the rated load is light. Overall, this paper provides comprehensive detail to detect the eccentricity faults using current, axial flux and instantaneous power signals simultaneously.
\end{abstract}

Index Terms-Condition Monitoring, Eccentricity, Frequency, Sensor, Spectrum.

\section{INTRODUCTION}

Eccentricity is defined as unbalanced air gap between the rotor and stator of an induction motor. It may be caused by incorrect position of the stator or rotor. Conventional techniques of detection of broken rotor bar and air-gap eccentricity were based on frequency domain analysis of voltage, current, and instantaneous input power. This paper uses all three signals namely current, voltage and instantaneous power simultaneously and thus allows better detection of air gap eccentricity. A comprehensive survey of the existing condition monitoring methods of air-gap eccentricity detection is presented in [1]. Both static and mixed eccentricities are investigated by measuring only flux in [2]. A current spectrum of an induction machine under eccentric air gap fault is used to monitor and detect eccentricity [3]. Wavelet analysis is another useful method for detecting air gap eccentricity [4]. The negative sequence components in supply currents due to the presence of a static eccentricity are proposed as a fault indicator. By monitoring the magnitude of negative sequence components, one could determine the degree of static

Department of Electrical Engineering

'COMSATS Institute of Information Technology, Lahore, Pakistan

${ }^{2}$ University of South Asia, Lahore, Pakistan

Email: "drintesarahmad@ciitlahore.edu.pk,"sshuja@ciitlahore.edu.pk

Knob 1

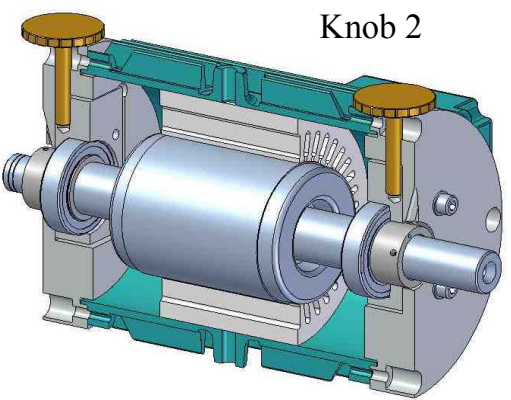

Figure 1. Test motor with two rotational knobs that are used to create unequal air-gap between stator and rotor.

eccentricity in the presence of a mixed eccentricity [5]. A comprehensive experimental study investigates the estimation of static eccentricity severity with commonly used eccentricity fault indicators under a wide range of loaded conditions [6].

In static air gap eccentricity, the angular position of the minimal radial air gap length is fixed in space; this may also be due to ovality of the stator core or incorrect positioning of the rotor or stator [7, 8]. Fig. 1 shows some modification made for the induction motor under test conditions. This motor serves as the basis of analyzing the fault frequency sideband components under static eccentricity faults. In this set-up the position of the minimal radial air-gap length was fixed in space. It is well understood that the incorrect positioning of the rotor or stator for any reason may cause static air-gap eccentricity to emerge. Therefore to create a state of air gap eccentricity, two rotational knobs one at driving-end (DE) of the motor and another one at nondriving-end (NDE) of the motor were used. By rotating these knobs in an upward or downward direction the position of minimal air gap length in space is altered to create an air gap eccentricity. The total air-gap between the rotor and stator of a machine is $0.8 \mathrm{~mm}(0.4 \mathrm{~mm}$ on both sides). Different combinations are used to analyze the eccentricity fault frequencies from current, flux and instantaneous power spectra under different load tests and compare the results to a healthy motor.

The different levels of eccentricity to be considered are as follows:

Non-driving and driving-end is at $0.1 \mathrm{~mm}$ and nondriving-end and non-driving-end is at $0.3 \mathrm{~mm}$ respectively.

TABLE I. DIFFERENT COMBINATIONS OF AIRGAP ECCENTRICITY DEVELOPED IN THE MACHINE TO STUDY THE SEVERITY OF ECCENTRICITY FAULTS UNDER DIFFERENT LOADING CONDITIONS.

\begin{tabular}{|c|c|c|c|c|c|}
\hline Airgap & \multicolumn{5}{|c|}{ Driving-End (mm) } \\
\hline \multirow{2}{*}{$\begin{array}{c}* \\
\text { Z }\end{array}$} & & +0 & +0.1 & +0.2 & +0.3 \\
\hline & -0.1 & $\mathrm{X}$ & $\mathrm{X}$ & $\mathrm{X}$ & $\mathrm{X}$ \\
\hline
\end{tabular}




\begin{tabular}{|c|c|c|c|c|c|}
\hline & -0.2 & $\mathrm{X}$ & $\mathrm{X}$ & $\mathrm{X}$ & $\mathrm{X}$ \\
\cline { 2 - 6 } & -0.3 & $\mathrm{X}$ & $\mathrm{X}$ & $\mathrm{X}$ & $\mathrm{X}$ \\
\hline
\end{tabular}

Non-driving-end is fixing at $0.1 \mathrm{~mm}$ (downward) and driving-end is at $0.2 \mathrm{~mm}$ and $0.3 \mathrm{~mm}$ upward (direction).

Non driving-end is fixing at $0.3 \mathrm{~mm}$ (downward) and driving-end is at $0.2 \mathrm{~mm}$ and $0.3 \mathrm{~mm}$ (upward) direction.

To detect eccentricity faults using the frequency spectrum of current, flux and instantaneous power signal, combinations of the air gap at both driving-end and at nondriving-end under different loading conditions will be used as given in Table 1.

\section{CONDITION MONITORING SYSTEM}

On-line condition monitoring means to assess the actual condition of a machine using measurements taken whilst the machine is operating. A block diagram of a condition monitoring system is shown in Fig. 2. In this stage, all the related terms used in condition monitoring during this study is described briefly. An extensive series of tests on a set of identical motors with a range of faults and under a variety of load conditions as mentioned in block (A) is performed. These faults affect the symmetry of the machine and as a result produce characteristic fault frequencies in the different types of sensors, shown in block (B).

In block (C), the data acquisition system is used to record the sensor signals in digital form. These signals can be analyzed by different digital signal processing techniques to extract features, which are sensitive to the presence of faults. Some of the common techniques are shown in block (D) [9].

In next step, the characteristic fault frequencies of different faults are detected from the processed signals. In this study, a customized MATLAB program is developed to detect these fault frequencies efficiently as shown in block (E).

Finally, in block $(F)$, the fault frequency characteristics that relate to different fault conditions are analyzed in the different sensor signals, (voltage, current, flux, and instantaneous power). This helps to better understand the relationship between fault type and severity versus the amplitudes of the characteristic fault frequencies.

\section{EXPERIMENTAL SETUP}

The experimental work was conducted using the test rig and data acquisition system as shown in Fig. 3. The tests

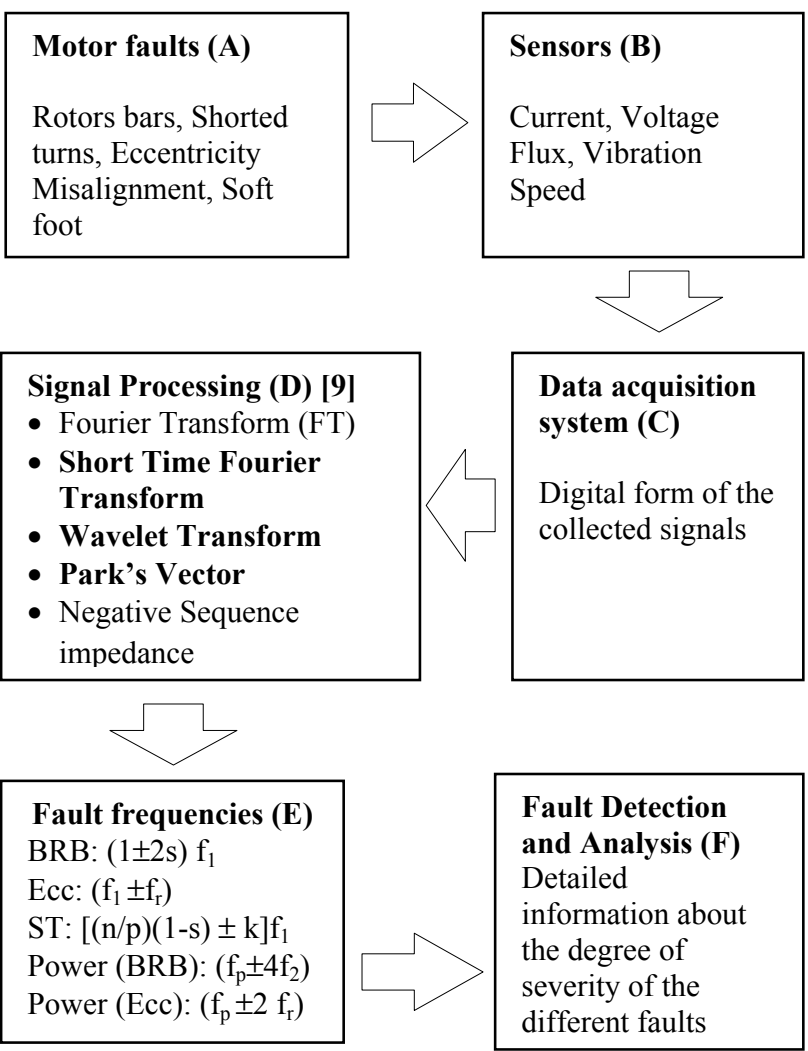

Figure 2. Condition monitoring system for baseline analysis.

were conducted on a set of healthy and faulty three-phase induction motors (T-DF100LA, manufactured by Brook Crompton), which were loaded by a dynamometer consisting of a $5 \mathrm{~kW}$ separately excited DC machine, using a variable-resistance bank. The detailed technical specifications of the induction motors under test are shown in Table 2.

The induction motor under test was mechanically coupled to a separately excited DC machine, which was loaded using a variable-resistance bank. The wiring diagram of the $\mathrm{AC}$ and DC systems is shown in Fig. 4. The specifications of the DC machine used as a load in the testing are shown in Table 3.

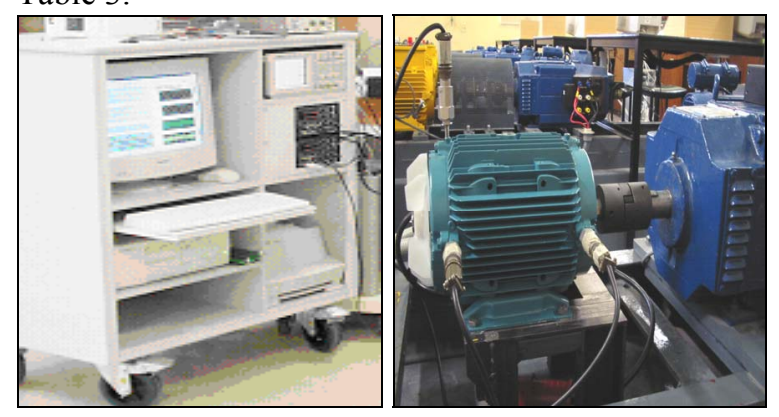

Figure 3. Data-acquisition hardware (left) and motor/load test set-up (right).

TABLE II TECHNICAL INFORMATION FOR THE INDUCTION MOTORS USED IN THE TESTING

\begin{tabular}{|c|c|}
\hline Performance Data & Value \\
\hline Rated voltage (V) & 415 \\
\hline
\end{tabular}




\begin{tabular}{|l|c|}
\hline Rated frequency $(\mathrm{Hz})$ & 50 \\
\hline Rated current (A) & 4.8 \\
\hline Power (kW) & 2.2 \\
\hline Number of Poles & 4 \\
\hline Rated speed (rpm) & 1415 \\
\hline Rated torque (Nm) & 14.8 \\
\hline Number of rotor slots & 32 \\
\hline Power factor at full-load & 0.81 \\
\hline
\end{tabular}

TABLE III TECHNICAL INFORMATION ABOUT THE DC MACHINE USED IN THE TESTING

\begin{tabular}{|l|c|}
\hline \multicolumn{1}{|c|}{ Performance Data } & Value \\
\hline Rated shaft power $(\mathrm{kW})$ & 5.5 \\
\hline Rated speed (rpm) & 1500 \\
\hline Rated armature voltage (V) & 220 \\
\hline Rated armature current (A) & 28 \\
\hline Rated field current (A) & 0.65 \\
\hline
\end{tabular}

During the tests, the two current sensors for line currents, two voltage sensors for line voltages, one axial leakage flux sensor, two vibration sensors: drive-end horizontal (DEH) and drive-end vertical (DEV), and one speed sensor that is directly connected to the shaft of motor, were used.

The position of the sensors in the test arrangement is shown in Fig. 4. The axial leakage flux measurement was taken with a circular search coil of comparable diameter to the motor, which is mounted concentrically with the shaft on the rear of motor. For reliability and consistency of the flux measurements, a fixed position for the flux coil is defined.

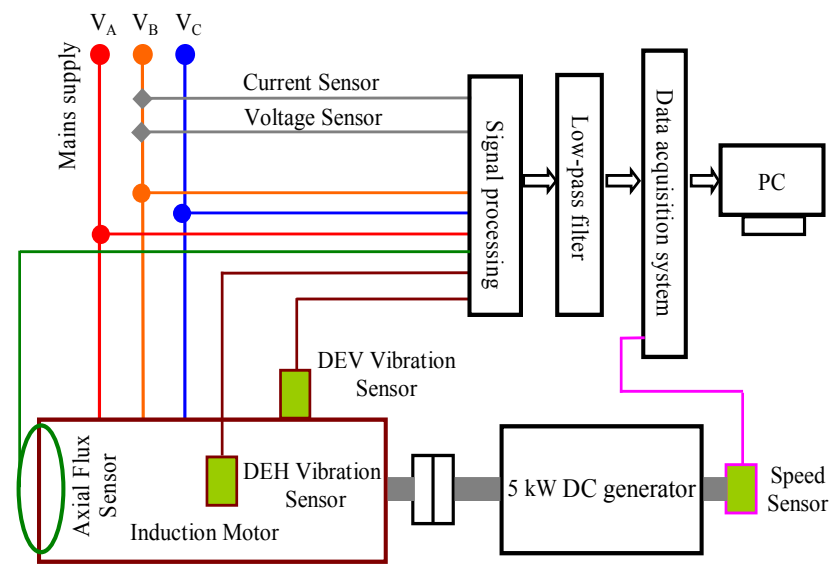

Figure 4. The block diagram of the test set-up including the positions of the sensors [8].

The vibration sensors were screw-mounted to the motor housing to achieve the highest measurement bandwidth. Measurements of the input stator currents and voltages were taken using a custom-built measurement box that was located between the auto-transformer and the test motor.

The speed sensor was directly attached to the motor shaft to obtain the running speed of motor as shown in Fig. 4.

The analog signals from the sensors are passed through low-pass filters to remove any high frequency components that may cause aliasing. This is performed by an 8 channel, 8th order Butterworth analog low-ass filter unit with selectable cut-off frequencies of $100 \mathrm{~Hz}$ for the $400 \mathrm{~Hz}$ sampling frequency and $2 \mathrm{kHz}$ for the $8 \mathrm{kHz}$ sampling frequency. Table 4 illustrates the specifications of the sensors used for measurements in this study.

The data-acquisition software used for data collection was written using Lab VIEW. The principal aim of this software is to collect the data, display it and save it for further analysis. The graphical user interface of the software is shown in Fig. 5. For this study, a custom written program developed in MATLAB is used to identify the fault frequencies from the current, flux and instantaneous power spectra.

There are number of factors which can affect the accuracy of the measurements. Some of these factors are the rigidity of the foundation of the test motor and the accuracy of the shaft alignment. The motors must have a firm and rigid foundation to eliminate soft foot problems and to reduce vibration [10]. There are number of cost benefits of precision alignment. It can help to reduce operating costs by reducing energy costs. Precision alignment also results in increased maintenance savings through reduced parts consumption and reduced overtime. Typically, a precision alignment of machines can reduce the energy costs from 3 to $10 \%$. A recent study showed that even small amount of misalignment could significantly reduce bearing life. To eliminate alignment faults, the motors were installed on the test rig using a precision laser alignment tool and a torque wrench (Norbar set at the recommended value of $35 \mathrm{Nm}$ for ideal mounting) as these two tools were used to ensure the accuracy and the repeatability of the mounting as well as to introduce the misalignment faults in machines.

TABLE IV SENSOR SPECIFICATIONS

\begin{tabular}{|l|l|l|l|l|}
\hline Signal & Device & $\begin{array}{l}\text { Band } \\
\text { width }\end{array}$ & $\begin{array}{l}\text { Input } \\
\text { Range }\end{array}$ & $\begin{array}{l}\text { Output } \\
\text { Gain }\end{array}$ \\
\hline Voltage & $\begin{array}{l}\text { Differential } \\
\text { Amplifier }\end{array}$ & $30 \mathrm{~Hz}$ & $\pm 600 \mathrm{~V}$ & $\begin{array}{l}100 \mathrm{~V}=1 \\
\mathrm{~V}\end{array}$ \\
\hline Current & $\begin{array}{l}\text { Hall-effect } \\
\text { sensor }\end{array}$ & $\begin{array}{l}50 \\
\mathrm{KHz}\end{array}$ & $\pm 10 \mathrm{~A}$ & $\begin{array}{l}10 \mathrm{~A}=1 \\
\mathrm{~V}\end{array}$ \\
\hline Flux & $\begin{array}{l}100 \text { turns } \\
\text { search coil }\end{array}$ & $\begin{array}{l}10 \\
\mathrm{kHz}\end{array}$ & $\pm 1 \mathrm{~V}$ & - \\
\hline Vibration & $\begin{array}{l}\text { Piezoelectric } \\
\text { Accelerometer }\end{array}$ & $\begin{array}{l}20 \\
\mathrm{Khz}\end{array}$ & $\pm 2 \mathrm{~g}$ & $1 \mathrm{~g}=1 \mathrm{~V}$ \\
\hline Speed & Tacho-meter & - & - & - \\
\hline
\end{tabular}




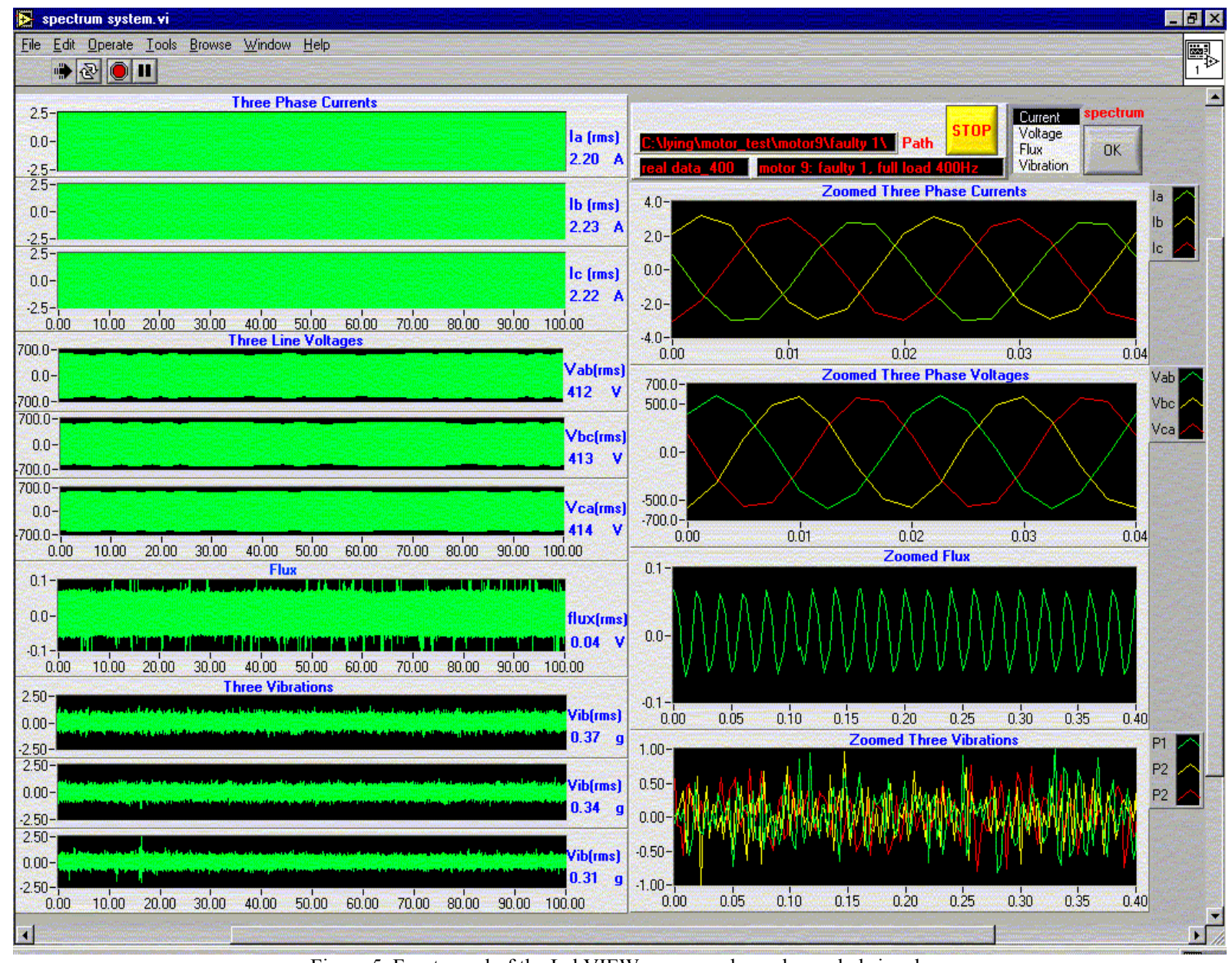

Figure 5. Front panel of the LabVIEW program showed sampled signals

During the measurements the sensors were sampled simultaneously and two different sampling rates used in this study are as follows:

Low-frequency measurement with a $400 \mathrm{~Hz}$ sampling frequency that gives a Nyquist frequency of $200 \mathrm{~Hz}$, and 100 second sampling time, which allow very highresolution frequency analysis $(40,000$ data points, $0.01 \mathrm{~Hz}$ resolution).

TABLE V SUMMARY OF SENSOR SIGNAL SAMPLING INFORMATION

\begin{tabular}{|l|l|l|}
\hline & $\begin{array}{l}\text { Low } \\
\text { Frequency } \\
\text { Sampling }\end{array}$ & $\begin{array}{l}\text { High } \\
\text { Frequency } \\
\text { Sampling }\end{array}$ \\
\hline Sampling Frequency & $400 \mathrm{~Hz}$ & $8 \mathrm{KHz}$ \\
\hline Total Sampling Time (T) & $100 \mathrm{sec}$ & $5 \mathrm{sec}$ \\
\hline $\begin{array}{l}\text { Frequency Resolution } \\
\Delta \mathrm{f}=(1 / \mathrm{T})\end{array}$ & $0.01 \mathrm{~Hz}$ & $0.2 \mathrm{~Hz}$ \\
\hline $\begin{array}{l}\text { Total number of points in } \\
\text { record LR =fs/ } \Delta \mathrm{f}\end{array}$ & 40,000 & 40,000 \\
\hline Nyquist Frequency & $200 \mathrm{~Hz}$ & $4 \mathrm{KHz}$ \\
\hline Cutt-off Frequency & $100 \mathrm{~Hz}$ & $2 \mathrm{KHz}$ \\
\hline
\end{tabular}

High-frequency measurement at $8000 \mathrm{~Hz}$ sampling frequency with a sampling time of 5 seconds (40,000 data points, $0.2 \mathrm{~Hz}$ resolution).

The parameters of the two sampling rates, achieved frequency resolution, frequency range, setting of antialiasing filter and hence useable frequency ranges of each sampling rate used for experiments are illustrated in Table 5.

\section{Method To Detect Eccentricity Faults Using DiFFERENT SENSOR SigNALS}

Variations in the fault frequencies were used to locate different faults in induction machines. Few authors [11, 12] suggested eccentricity faults produce fault frequency components at $\left(\mathrm{f}_{1} \pm \mathrm{fr}_{\mathrm{f}}\right)$ and at $\mathrm{f}_{1}[(\mathrm{R} / \mathrm{p})(1-\mathrm{s}) \pm \mathrm{k}]$ in the current, flux spectra and modified frequency components at ( $\mathrm{f} \pm 2 \mathrm{fr}$ ) and at $\mathrm{f}_{1}[(\mathrm{R} / \mathrm{p})(1-\mathrm{s}) \pm \mathrm{k}]$ were used in the instantaneous power spectra. These components are centered on the rotor slot passing frequency $\mathrm{R} f \mathrm{r}$ ( $\mathrm{R}$ is the number of rotor bars) and integers $\mathrm{k}$ for different values $\pm 1, \pm 3$, and \pm 5 . In this paper fault frequency components at $\left(\mathrm{f}_{1} \pm \mathrm{fr}\right)$ in the current and flux spectra and at ( $\mathrm{fp} \pm 2 \mathrm{fr})$ were in the instantaneous power were considered. However, accurate detection of eccentricity faults heavily depends on the accurate measurement of slip frequency $s f_{1}$ and rotor frequency fr. Therefore, it is important to understand how to locate the correct eccentricity fault frequency peaks in the frequency spectrum of current, flux and instantaneous power signals.

To understand the different steps involved in locating eccentricity faults in the frequency spectrum using fault frequencies from different signals, a healthy motor $(2.2 \mathrm{~kW})$ under full-load test is consider to identify the correct fault frequency peaks in the current spectrum with the help of slip frequency sfi measured from flux spectra and slot passing frequency, which is measured from vibration spectra. It should be noted that a similar method is used to 
detect the faults using flux and instantaneous power spectra. Measured supply frequency $f_{1}$ from current spectra, which is in this case, is $50 \mathrm{~Hz}$ as shown in Fig. 6.

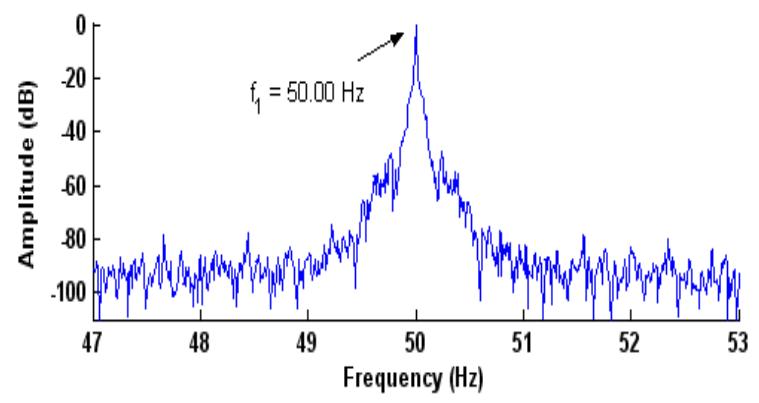

Figure 6. Current spectra showing the supply frequency.

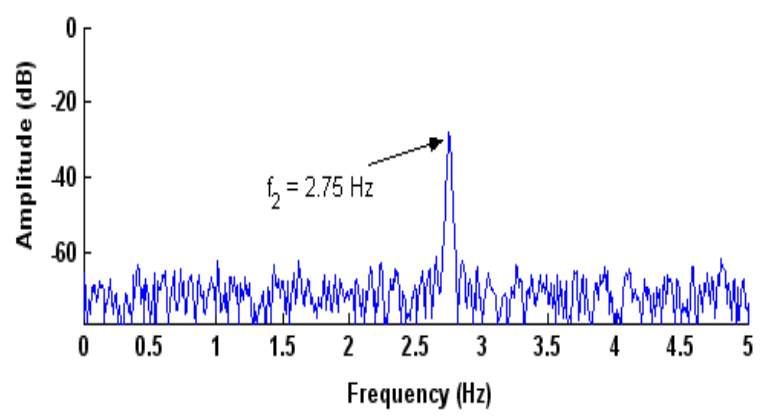

Figure 7. Flux spectra showing the slip frequency.

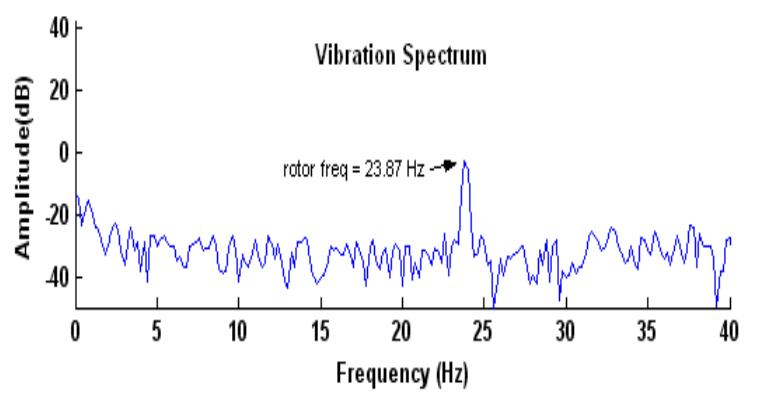

Figure 8. Frequency spectrum of vibration signal showing the rotor frequency.
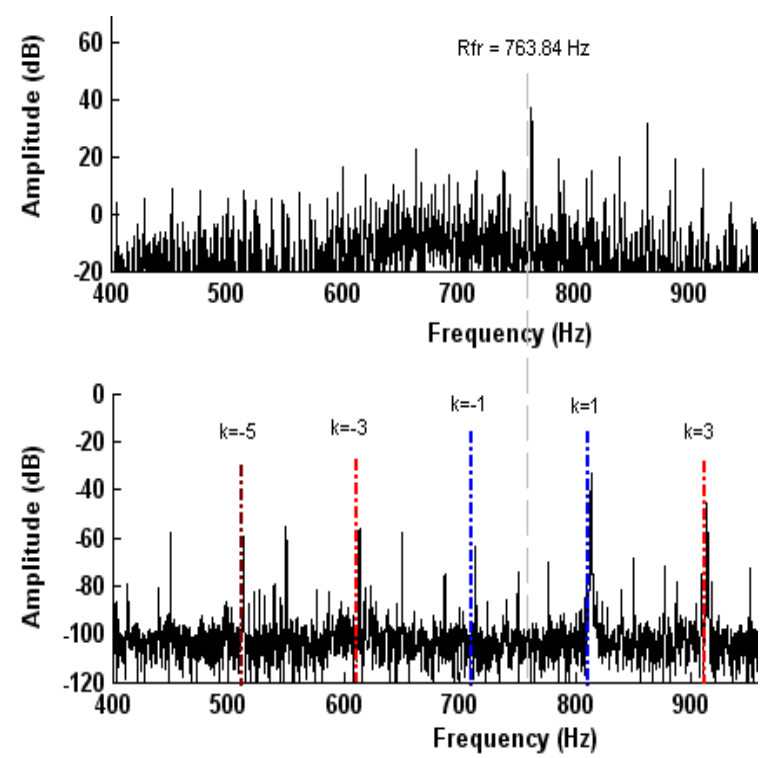

Figure 9. Frequency spectrum of vibration signal (top) showing slot passing frequency and current spectrum (bottom) showing eccentricity fault frequency peaks.

Measured slip frequency $\mathrm{sf}_{1}=\mathrm{f}_{2}$ from flux spectra, which is $2.29 \mathrm{~Hz}$ as shown in Fig. 7.
Measured rotor frequency fr from vibration spectra as shown in Fig. 8, which is $23.87 \mathrm{~Hz}$.

Measured slot passing frequency using vibration spectra, which is $\mathrm{Rfr}=763.84 \mathrm{~Hz}$ as shown in Fig. 9(top). Measured fault frequencies related peaks and their respective sideband amplitudes for different values of $\mathrm{k}$ in the current spectra are shown in Fig. 9 (bottom), dashed lines represent the fault frequencies peaks.

To Confirm the accuracy and reliability of fault frequency components detecting the eccentricity faults using current, flux and instantaneous power signals, these measured values of rotor and slot passing frequencies from current, flux and instantaneous power spectra should be same or very close to the calculated values of the rotor and slot passing frequencies. It is important to calculate the rotor and slot passing frequencies of the same healthy motor. Checking the utilization of fault frequency peaks may lead to eccentricity faults being detected in the frequency spectrum of current, flux and instantaneous power signals. Calculation steps are as follows:

To calculate the rotor frequency from $\mathrm{fr}=\left(\mathrm{f}_{1}-\mathrm{sf} 1\right) / \mathrm{p}$, the slip frequency sf1 is calculated from $\mathrm{f}_{2}=\left(\mathrm{N}_{\mathrm{s}}-\mathrm{Nr}\right) / \mathrm{p} \mathrm{Hz}$, which is $23.87 \mathrm{~Hz}$ than calculate the slot passing frequency by using the relationship $\mathrm{Rfr}$ that is equal to $763.84 \mathrm{~Hz}$ (when the number of rotor bars $\mathrm{R}=32$ ).

It has been concluded from the results, that there is almost no difference between the measured and calculated values of rotor and slot passing frequencies, which confirm the accuracy and reliability of characteristics fault frequency components to detect the eccentricity faults using current, flux and instantaneous power spectra. The following section, we will consider the current, flux and instantaneous power signals to detect the eccentricity faults under different levels of loading.

\section{DETECTION Of ECCENTRICITY FAULT USING DiFFERENT SENSOR SIGNALS}

The comparison of eccentricity sideband components ( $f_{1-}$ fr) for healthy and faulty motor with airgap of $(-0.1 \mathrm{~mm}$ at $\mathrm{NDE}$ and $+0.3 \mathrm{~mm}$ at DE) at $30 \%$ load and at full-load for current, flux and instantaneous power spectra is shown in Fig. 10-18 respectively. The circles indicate the eccentricity sideband components.

It can be concluded from Fig. 10, that, variation in the amplitudes of sideband components of a faulty motor at $30 \%$ load is more when compared to the faulty motor at full-load. In addition, amplitudes of the fault frequencies decreased as the load on the motor increase. The high amplitudes of fault frequency peaks can be helpful in assessing the motor's condition. The noise level is about $90 \mathrm{~dB}$.

It has been observed from flux spectra in Fig. 12 and Fig. 13; variations in the amplitudes of the eccentricity fault frequency components of a faulty motor at $30 \%$ load and at full-load are higher than the current spectrum at the same level of loading. The noise level is $(-70 \mathrm{~dB})$, which is high. 

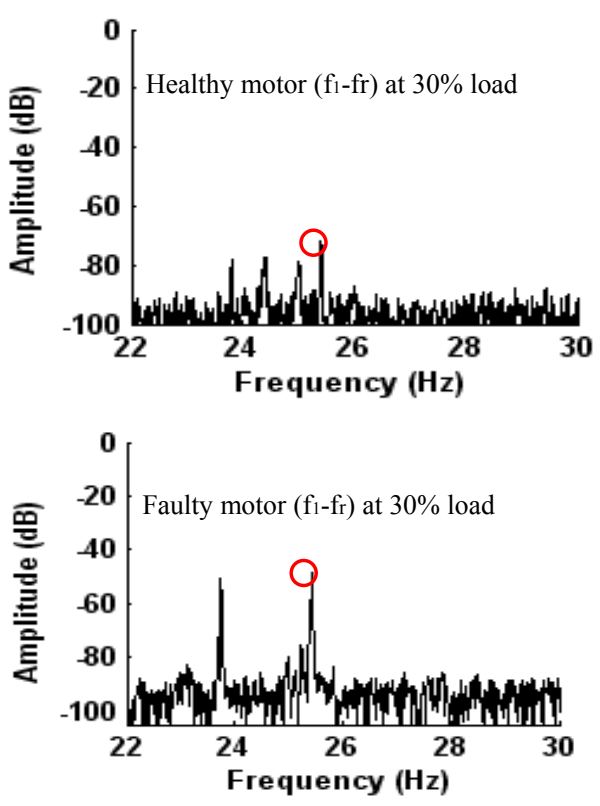

Figure 10: Current spectrum from a healthy and a faulty machine at $30 \%$ load.
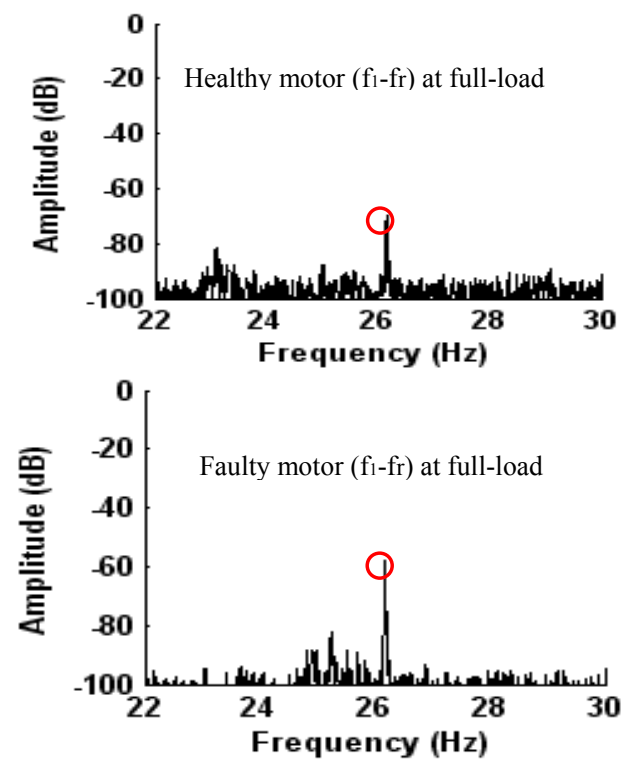

Figure 11: Current spectrum from a healthy and a faulty machine at fullload.
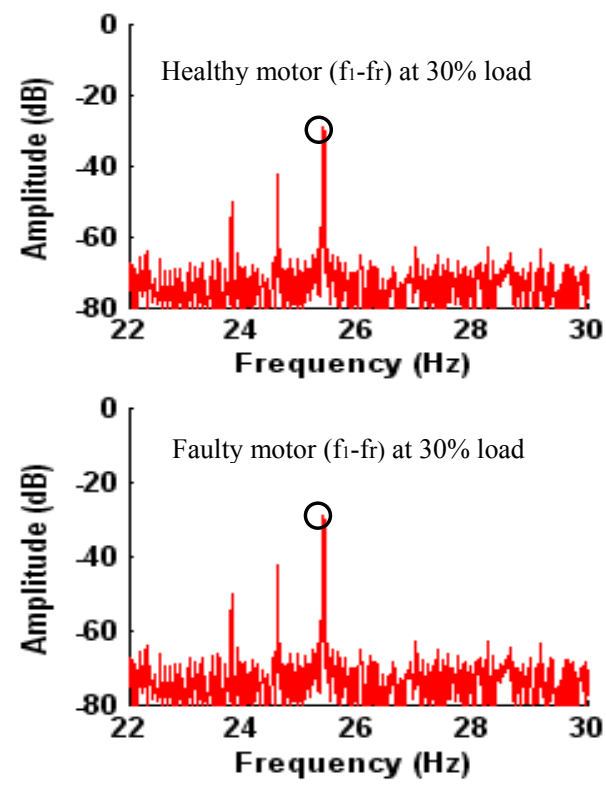

Figure 12: Flux spectrum from a healthy and a faulty machine at 30\% load.

The magnitude of the fault frequency obtained from the instantaneous power spectra is shown in Fig. 14 and 15 that the changes in the amplitudes of positive sideband component of faulty motor at $30 \%$ load is slightly higher. However, fault frequency components were not clearly visible in the spectrum when compared with current and flux spectra.
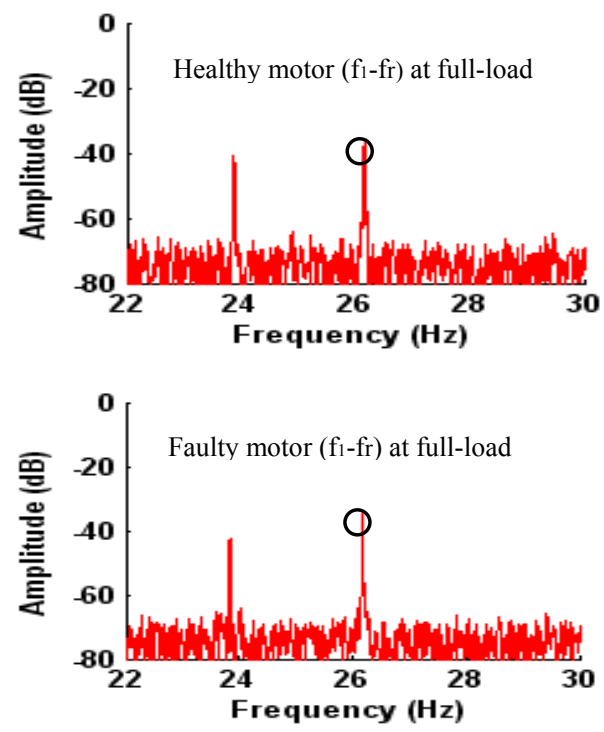

Figure 13: Flux spectrum from a healthy and a faulty machine at full-load.
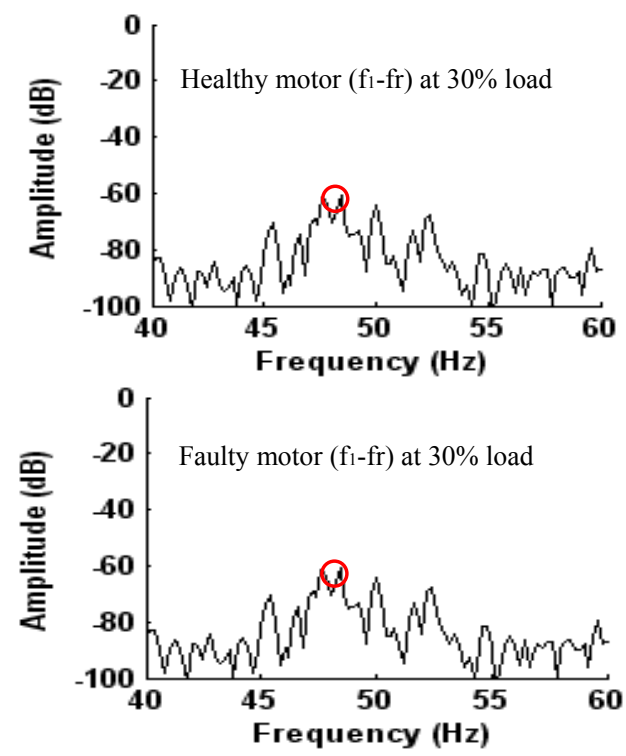

Figure 14: Instantaneous Power spectra from a healthy and a faulty machine at $30 \%$ load.

\section{ANALYSis Of Sideband COMPONENTS To Detect ECCENTRICITY FAULT USING CURRENT, FLUX AND INSTANTANEOUS POWER SIGNALS}

The variations in the amplitude of eccentricity fault frequency components $\left(f_{1} \pm f_{r}\right)$ between the healthy motor (zero eccentricity) and faulty motor with eccentricity $(>0.05$ $\mathrm{mm})$ were found to be more than $(20 \mathrm{~dB})$ in the current spectra, which is significant and clearly visible at light load as shown in Fig. 16. The results indicate the consistent increase in the fault frequency components with the increase 
in eccentricity levels, making it easier to
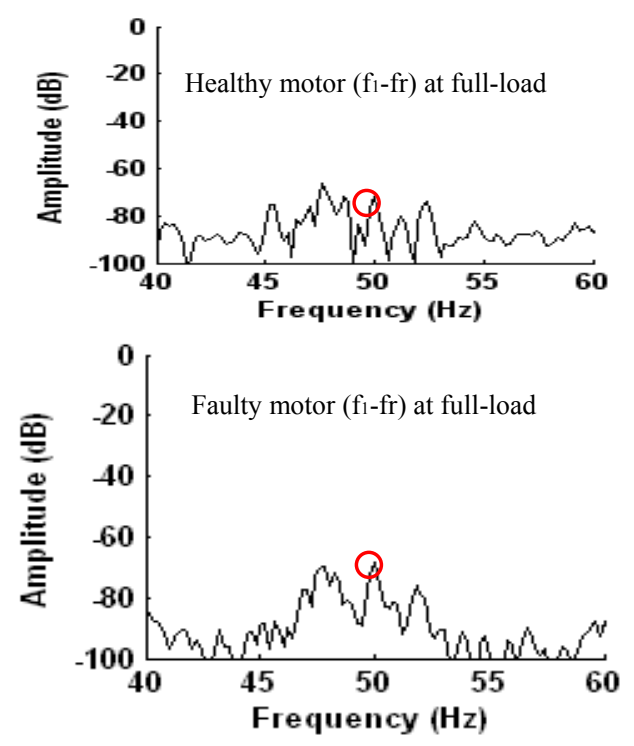

Figure 15: Instantaneous Power spectra from a healthy and a faulty machine at full-load.
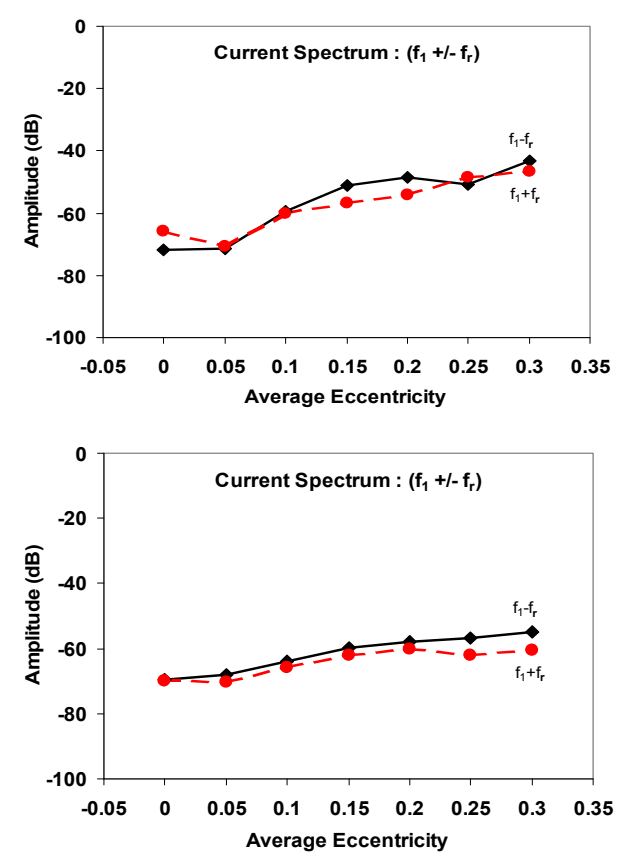

Figure 16: Variations in the sideband amplitudes versus average eccentricity at $30 \%$ (top) and at full-load (bottom) between the healthy and faulty motors from current spectrum

differentiate between healthy and faulty motors using current spectrum.

Fig. 16 also shows that the variations in the sideband amplitudes between the healthy and faulty motors at fullload are consistent when the air gap is $(>0.05 \mathrm{~mm})$. However, a slight declined in the variations of sideband amplitudes was found between the healthy and faulty motors under full-load test conditions when compared to the variations for the same motors running at $30 \%$ load.
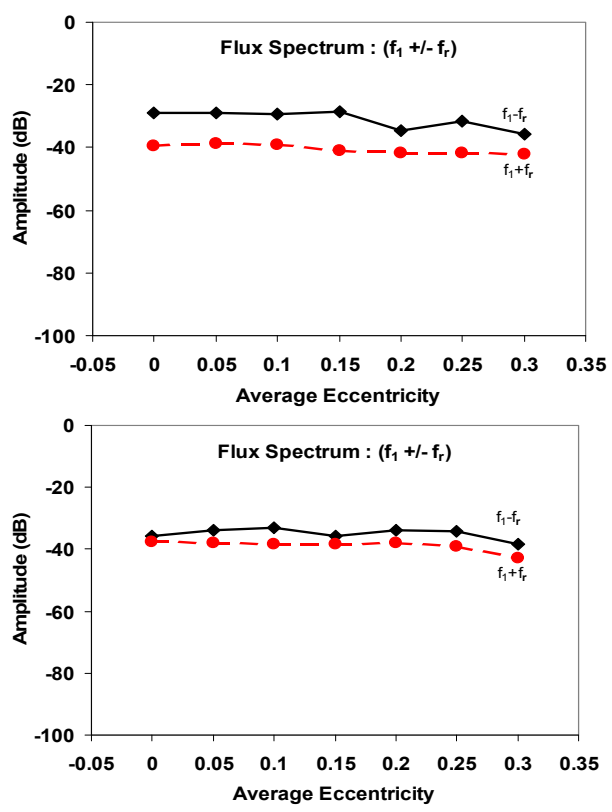

Figure 17: Variations in the sideband amplitudes versus average eccentricity at $30 \%$ (top) and at full-load (bottom) between the healthy and faulty motors from flux spectrum

The results from current spectra present that the eccentricity fault frequency components are suitable to detect eccentricity faults at any level of eccentricity under any level of loading conditions (specifically at light loads).

Eccentricity fault frequency components $\left(f_{1} \pm f r\right)$ from the flux spectrum for 30\% and at full-load are shown in Fig. 17.

It shows that variations in the sideband components of the faulty motors were not affected at $30 \%$ load compared to the top illustration in the Fig. 17. However, at full-load slight increase in the fault frequency components at the different levels of average eccentricity were found when reference was made to the faulty motor at $30 \%$ load as shown in Fig. 17 (bottom).

It is evident from test results that the eccentricity fault frequency components are more detectable in the current spectrum. Furthermore, the results obtained from the flux spectrum clearly indicate that the variations in the sideband amplitudes are not significant at both light and heavy loads. Therefore, it is not a suitable medium to detect eccentricity faults using fault frequency components ( $\left.f_{1} \pm f r\right)$.

Amplitude of fault frequency components ( $\mathrm{f} p \pm 2 \mathrm{fr}$ ) versus average eccentricity is shown in Fig. 18 at 30\% load and at full-load. Variations in the amplitudes of components of faulty motors at different level of eccentricity were found to be significant.

The results showed that the maximum variations in the negative sideband component occurred when the levels of air gap eccentricity are $0.05,0.2$ and $0.25 \mathrm{~mm}$. For the positive sideband component the maximum variations were found when the levels of air gap eccentricity were 0.2 and $0.3 \mathrm{~mm}$. 

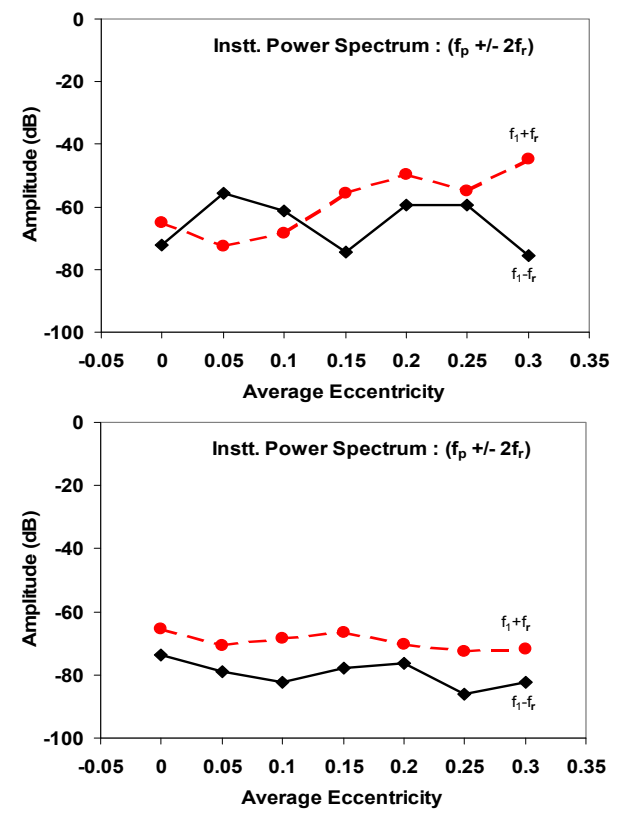

Figure 18: Variations in the sideband amplitudes versus average eccentricity at $30 \%$ (top) and at full-load (bottom) between the healthy and faulty motors from instantaneous power spectrum

However, the result presented in Fig. 18, indicate that any variation in the sideband components was only significant when the degree of eccentricity was $0.25 \mathrm{~mm}$ at full-load. It can be concluded from the results, that instantaneous power is suitable for detecting the eccentricity fault at light loads. Since the instantaneous power provides good results to detect the eccentricity faults. It can therefore be assumed that it is necessary to check its variability in healthy motors.

\section{CONCLUSION}

Previous studies used variations in the amplitudes of the fault frequency components $\left(f_{1} \pm f r\right)$, between the healthy motor and faulty motor versus $\%$ of rated load of the motor to detect eccentricity faults.

In comparison, this work examines variations in the amplitudes of the fault frequency components between the healthy motor and faulty motor versus average eccentricity level over a wide range of loading conditions, which is calculated by using formula "Average Eccentricity" $=[(\mathrm{E} 1+\mathrm{E} 2) / 2]$, which is introduced in this work. The selected range for average eccentricity level is from 0 to 0.3 $\mathrm{mm}$ (because the maximum airgap between the stator and rotor is $0.4 \mathrm{~mm}$ at both sides of the machine).

The variations in the sideband amplitudes $\left(f_{1} \pm f r\right)$ between the healthy and faulty motors showed good features over the full range of load tested to detect the eccentricity fault in the current spectra. The results from instantaneous power indicate that the variations between the healthy and faulty motors are significant when the level of average eccentricity is $0.2 \mathrm{~mm}$ and the minimum rated load is $30 \%$. Therefore, instantaneous power is useful but less effective due to random changes in the sideband amplitudes. However, flux signal alone is not suitable for detecting eccentricity faults for the same. So the presented technique is a promising approach when it comes to mitigate the above mentioned problems.

\section{REFERENCES}

[1] Pinjia Zhang, Yi Du, Habetler, T.G., Bin Lu, "A Survey of Condition Monitoring and Protection Methods for Medium Voltage Induction Motors," IEEE Energy Conversion Congress and Exposition, 2009, ISBN: 978-1-4244-2893-9, pp. 3165 - 3174.

[2] Negrea, M., Jover, P., Arkkio, A., "Electromagnetic Flux-Based Condition Monitoring for Electrical Machines," 5th IEEE International Symposium on Diagnostics for Electric Machines, Power Electronics and Drives September 2005 pp. 1 - 6.

[3] Schoen, R.R., Habetler, T.G., "Effects of Time-Varying Loads on Rotor Fault Detection in Induction Machines," IEEE Transactions on Industry Applications, Vol. 31, No. 4, July/August 1995, pp. 900-906.

[4] Bin Lu, Paghda, M., "Induction Motor Rotor Fault Diagnosis using Wavelet Analysis of One-Cycle Average Power," $23^{\text {rd }}$ Annual Applied Power Electronics Conference and Exposition, 2008. ISBN: 978-1-4244-1873-2, pp. $1113-1118$.

[5] Long Wu, Bin Lu, Xianghui Huang, Habetler, T.G., Harley, R.G, "Improved Online Condition Monitoring Using Static EccentricityInduced Negative Sequence Current Information in Induction Machines," Industrial Electronics Society, 2005. IECON, 31st Annual Conference of IEEE, ISBN: 0-7803-9252-3, NOV 2005.

[6] Grieger, J., Supangat, R., Ertugrul, N., Soong, W.L., Gray, D.A., Hansen, C., "Estimation of Static Eccentricity Severity in Induction Motors for On-Line Condition Monitoring," Industry Applications Conference, 2006. $41^{\text {st }}$ IAS Annual Meeting. Conference Record of the 2006 IEEE, Vol. 5, Oct. 2006, pp 2312-2319.

[7] M. L. Sin, W. L. Soong and N. Ertugrul, "On - Line Condition Monitoring and Fault Diagnosis - A Survey" Australian Universities Power Engineering Conference, New Zealand, 2003.

[8] Ahmed, R. Supangat, J. Grieger, J, N. Ertugrul, and W.L Soong, "A Baseline Study for On-Line Condition Monitoring of Induction Machines", Australian Universities Power Engineering Conference, Brisbane, Australia, 2004.

[9] W. R. Finlay., "Troubleshooting motor problems," IEEE Trans. Ind. Applicat., vol. 30, pp. 1383-1397, Sept./Oct. 1994.

[10] I. Ahmed, N. Ertugrul, and W. L. Soong, "A Study on the Detection of Fault Frequencies for Condition Monitoring of Induction Machines," Australian Universities Power Engineering Conference Tasmania, Australia 2005.

[11] Ahmed, I., Ertugrul, N., and Soong, W. L. "A Study of Detection of Broken Rotor Bars in Induction Motors Using Instantaneous power", Energy and Power System International Conference 'EPS 2006' IASTED, Chiang Mai, Thailand, 28-30th March 2006.

S. F. Legowski et al, "Instantaneous power as a medium for the signature analysis of induction motors," IEEE Trans. Ind, Applicant, vol 32, pp. 904-909, July/Aug. 1996.

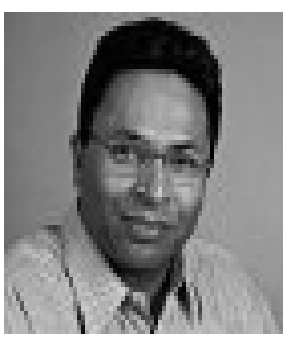

Dr. Intesar Ahmed received $\mathrm{PhD}$ Electrical Power Engineering from University of Adelaide and M.Sc. in Electrical Power Engineering from University of New South Wales, Sydney, Australia in 2007 and 1994 respectively. He has been involving in various research projects relating to

online condition monitoring of electrical machines, power electronics, and the eccentricity faults in machines via signal processing techniques.

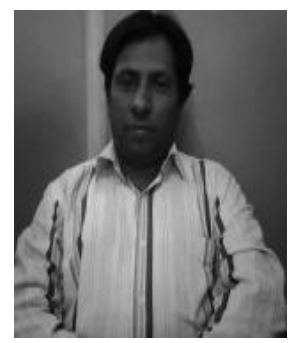

Manzar Ahmed received MS in Telecommunication from Asian Institute of Technology, Thailand and B.Sc. in Electrical Engineering from University of Engineering \& Technology, Lahore Pakistan in 2001 and 1991 respectively. His current research interests include electric machines and drives, electromagnetic computational and electromechanical actuation as well as techniques for energy savings. 


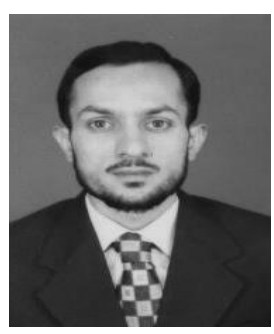

Kashif Imran completed M.Sc. in Electrical Engineering and B.Sc. in Electrical (POWER) Engineering from University of Engineering \& Technology, Lahore Pakistan in 2008 and 2006 respectively. His professional experience includes design of power distribution systems. His research deals

In Restructured Power Systems Simulation, Online Condition Monitoring and Power System Protection.

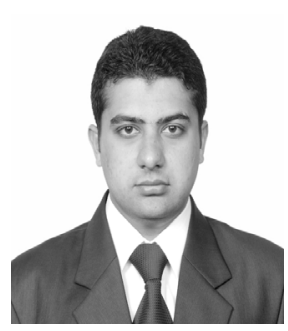

M. Shuja Khan received MS in Electronic Engineering from Ghulam Ishaq Khan Institute of Engineering. Science \& Technology, Swabi Pakistan and B.Sc. Electrical (POWER) Engineering from University of Engineering \& Technology, Lahore Pakistan in June 2009 and July 2007 respectively. His research interests include electric motor drives and MEMS design.

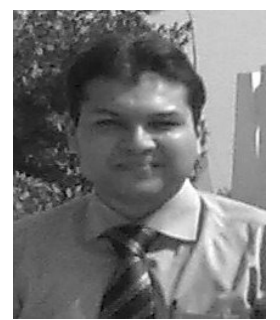

S. Junaid Akhtar received MS in Information and Communication Engineering from University of Leicester, England and BS in Computer Engineering from COMSATS Institute of Information Technology, Islamabad, Pakistan in 2007 and 2005 respectively. His research interests include digital signal processing for the detection of abrupt changes and fault diagnosis. 